\title{
Effect of chain length of self-assembled monolayers on adhesion force measurement by AFM
}

\author{
LI-YA GUO and YA-PU ZHAO* \\ State Key Laboratory of Nonlinear Mechanics (LNM), Institute of Mechanics, Chinese Academy of \\ Sciences, Beijing 100080, P.R. of China
}

Received in final form 25 May 2006

\begin{abstract}
It has been confirmed experimentally that the adhesion force measured between an atomic force microscope (AFM) tip and the self-assembled monolayers (SAMs) has a direct correlation with the chain length of SAMs, and that the adhesion force decreases with the increase of the chain length. In this paper, a theoretical model is put forward to calculate the adhesion force between the AFM tip and the SAMs by integrating the Lennard-Jones potential. The theoretical results are in good agreement with the existing experimental results.
\end{abstract}

Keywords: Quasi-continuum model; adhesion; SAMs; chain length; Lennard-Jones potential; van der Waals force; pull-off point.

\section{INTRODUCTION}

Microelectromechanical systems (MEMS) are rapidly being developed due to their low energy consumption, high integration, mass production and low unit cost. However, the large surface area-to-volume ratios raise serious adhesion and frictional problems to limit the widespread use of MEMS [1,2]. As a molecularlevel lubricant, self-assembled monolayers (SAMs) have attracted much attention [3-6] and have been demonstrated to be able to effectively reduce the friction and adhesion in MEMS [4, 7-9]. SAMs are ordered molecular assemblies formed by spontaneous adsorption of an active surfactant onto the surfaces of appropriate substrates, which consist of three building blocks: an active head group that binds strongly to the substrate, a terminal (end) group that constitutes the outer surface of the film, and an alkyl backbone chain that connects the head and terminal groups [5, 10,11]. As shown in Fig. 1 [4], each part of SAMs has a significant effect on

\footnotetext{
*To whom correspondence should be addressed. Tel.: (86-10)6265-8008. Fax: (86-10)6256-1284. E-mail: yzhao@lnm.imech.ac.cn
} 


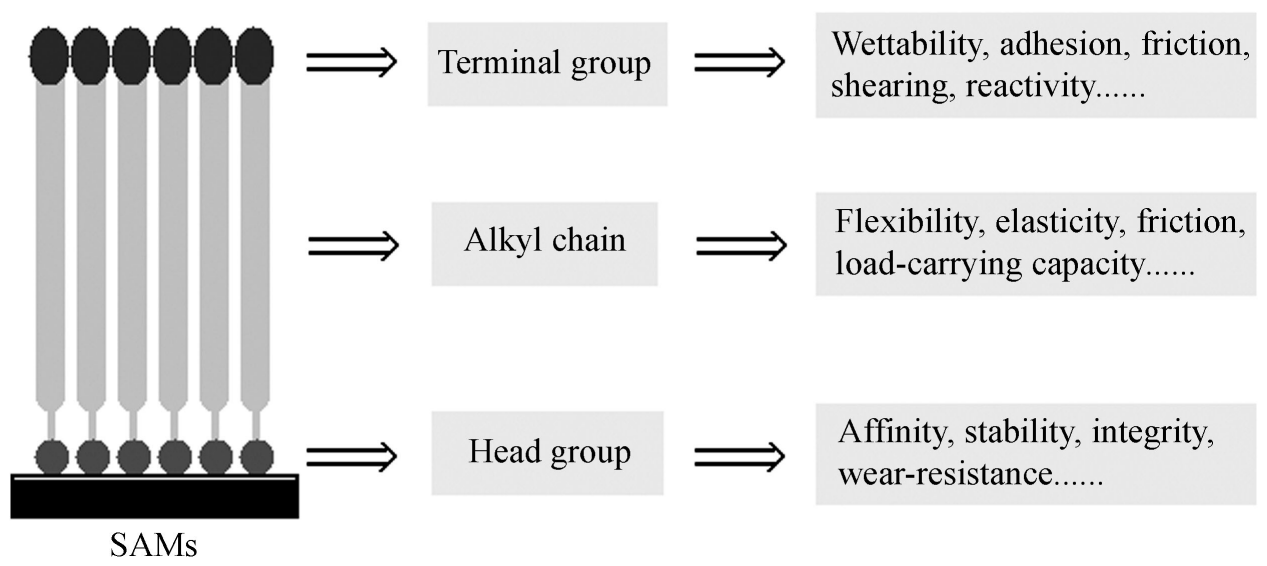

Figure 1. Schematic of a self-assembled monolayer and the effects of its different segments.

their tribological and mechanical properties. Relationship between the chemical structures and tribological properties of SAMs has been extensively studied to obtain boundary lubricants with good lubrication, high adhesion resistance and strong anti-wear ability [12-21]. For example, a novel self-assembled dual-layer film as a potential lubricant for MEMS was successfully designed and prepared by introducing hydrogen bonds and layer structure in the film, which not only could greatly reduce the friction and adhesion, but also possessed relatively high anti-wear ability [17].

Because of the large surface area-to-volume ratios of the micro-devices, adhesion might be the most serious tribological problem in MEMS for their operation. It is well known that the adhesion of SAMs is mainly controlled by the chemical characteristics of the terminal group and the environment, which has been studied extensively $[13,14,18,21]$. A representative study on the effect of terminal group on adhesion was done by Frisbie and co-workers [18, 21]. By using atomic force microscopy (AFM), they investigated adhesion force between the probe tip and sample surfaces both molecularly modified by SAMs with different terminal groups and found it to be in the order: $\mathrm{COOH} / \mathrm{COOH}>\mathrm{CH}_{3} / \mathrm{CH}_{3}>\mathrm{COOH} / \mathrm{CH}_{3}$. The influence of relative humidity on adhesion was studied in an environmentally controlled chamber at $22^{\circ} \mathrm{C}$ [19]. The results showed that for $\mathrm{Si}(111), \mathrm{Au}(111)$, DHBp (4,4'-dihydroxybiphenyl) and MHA (16-mercaptohexadecanoic acid thiol), the adhesion force increased with relative humidity; for BPT (1,1'-biphenyl-4-thiol) and BPTC (cross-linked BPT), the adhesion force increased only slightly with relative humidity when the relative humidity was higher than $40 \%$. Actually, in addition to the terminal group and the environment, the molecular chain-length also plays a significant role in the adhesion of SAMs. However, only a few experimental and molecular simulation works have been engaged in the studies of chain-length dependence of adhesion [20,22], although considerable amount of work has been done on chain-length dependence of friction $[12,14,23-25]$. By using molecular 
dynamics simulations, Chandross et al. reported that the adhesion force between two monolayers at the same separation distance decreases monotonically as the chain length increases from 6 to 18 carbon atoms [22].

As far as we know, no theoretical model for the chain-length dependence of adhesion of SAMs has been reported yet. In this paper, we attempt to establish a quasi-continuum model in which the AFM tip is considered as a small sphere and the SAMs molecules are treated as rigid rods without any degree of freedom; this model would help us to gain a deep insight into the effect of chain length on the adhesion force of SAMs. Since all previous publications on the subject have dealt with experimental study, our work attempts to establish a theoretical model. All the calculations are carried out in vacuum and the adhesion force between the AFM tip and the sample is obtained. Then we focus on the effect of chain length on the adhesion force. The molecular chain length, which determines the structural order of the films, has a significant influence on adhesion.

\section{THEORETICAL MODEL}

AFM is an effective and useful tool in investigating the adhesion force of SAMs, which is realized by obtaining a force-displacement curve (Fig. 2) and the pulloff force was defined as the adhesion force [25, 26]. The adhesion force between an AFM tip and a sample surface generally consists of the van der Waals (vdW) force, electrostatic force, chemical bonding force and capillary force [27]. For the monolayers with apolar terminal group (such as $\mathrm{CH}_{3}$ and $\mathrm{CF}_{3}$ ) in a vacuum environment, the capillary force can be eliminated and the contributions of electrostatic force or chemical bonding force can also be neglected. Thus, only the vdW force is considered in the present quasi-continuum model. Other hypotheses are also made: (1) the AFM tip is considered to be a small sphere; (2) the molecules in the SAMs are treated as rigid rods without any degree of freedom and to be vertical to the substrate [28] such a hypothesis may have a slight departure from reality in that the molecules in SAMs are tilted on the substrate surface at certain angles. However, for some kinds of SAMs, such as alkylsilanes on silicon surface and fatty acids on $\mathrm{Al}_{2} \mathrm{O}_{3}$ surface, the molecules are almost vertical to the substrate and there is only very little free volume among the molecules [5]. Thus, such hypothesis will not cause much error in our calculation. It should also be pointed out that only the effect of chain length on the adhesion force is considered in this proposed model, although many other factors (such as the terminal group of the SAMs and the environmental condition) could also have an important effect on the adhesion.

The vdW force exists between all surfaces in contact, which consists of three components and the corresponding potentials are Keesom potential, Debye potential and dispersion potential [25, 29]. Considering an AFM tip with radius $R$ and a monolayer with chain length $l$ as shown in Fig. 3, the intermolecular potential between the tip and sample is modeled by the Lennard-Jones (L-J) potential of 


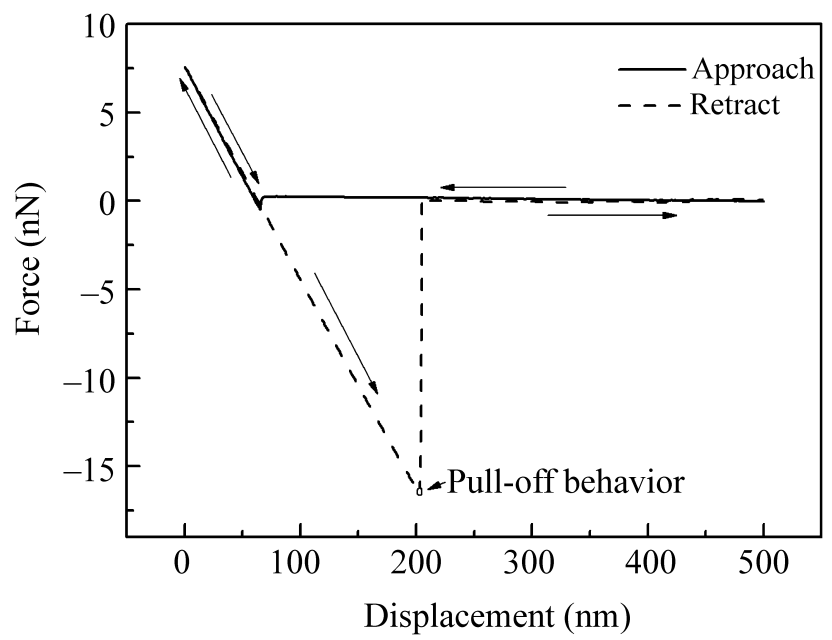

Figure 2. Force-displacement curves for silicon nitride tip and mica substrate.

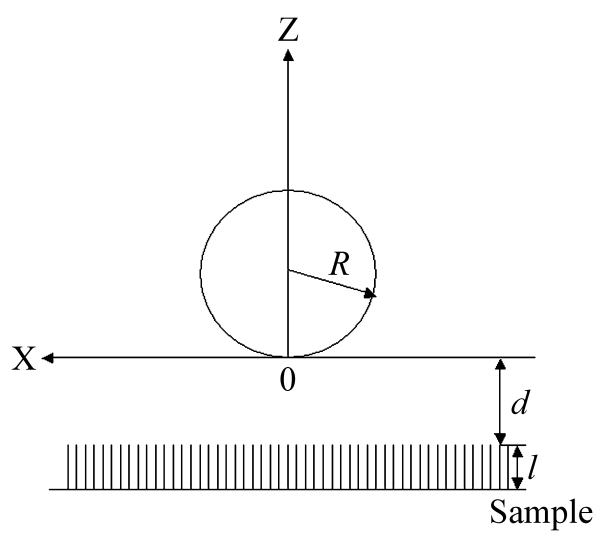

Figure 3. Tip-sample interaction model.

the form:

$$
e(r)=\frac{C r_{0}^{6}}{r^{12}}-\frac{C}{r^{6}},
$$

where the $r^{-12}$ term is related to the repulsive potential and the $r^{-6}$ term to attractive potential. $C$ is the constant in the atom-atom pair potential and $r$ is the distance between two molecules. The parameter $r_{0}$ can be considered as a characteristic length in intermolecular interaction, typically taken as $0.3 \mathrm{~nm}$. The L-J potential is an effective potential that describes the interaction between two uncharged molecules or atoms. It reflects the interactions in the united atom model such as a $\mathrm{CH}_{2}$ unit or even after integration it reflects the interaction between two segments, which consist of several $\mathrm{CH}_{2}$ units [10]. 
When calculating the potential between the tip and SAMs, we integrate the L-J potential first and obtain the function below, which represents the potential between an atom and the SAMs at a distance $z$ (see Appendix B)

$$
\Phi_{\mathrm{LJ}}=2 \pi \rho_{1}\left[\frac{C_{1} r_{0}^{6}}{90 z^{9}}-\frac{C_{1} r_{0}^{6}}{90(z+l)^{9}}+\frac{C_{1}}{12(z+l)^{3}}-\frac{C_{1}}{12 z^{3}}\right],
$$

where $\rho_{1}$ is the number density of molecules in the SAMs, $C_{1}$ is the interaction potential between the atom and the SAMs and $l$ is the length of SAM molecules.

With the assumption of additivity, the potential energy between the tip and SAMs at a distance $d$ will be the sum of interactions between all the molecules of the tip and SAMs. By integrating equation (2), the potential energy is given as:

$$
\begin{aligned}
W_{1}= & \int_{0}^{2 R} \frac{A_{1} r_{0}^{6}(2 R-z) z}{45(d+z)^{9}} \mathrm{~d} z-\int_{0}^{2 R} \frac{A_{1} r_{0}^{6}(2 R-z) z}{45(d+z+l)^{9}} \mathrm{~d} z+\int_{0}^{2 R} \frac{A_{1}(2 R-z) z}{6(d+z+l)^{3}} \mathrm{~d} z \\
& -\int_{0}^{2 R} \frac{A_{1}(2 R-z) z}{6(d+z)^{3}} \mathrm{~d} z
\end{aligned}
$$

where $A_{1}=\pi^{2} C_{1} \rho_{1} \mu$ is the Hamaker constant for the tip and SAMs, $\mu$ is the density of molecules of the tip and $R$ is the radius of the tip. Here the vdW force is considered to be additive and non-retarded. For $R \gg d$, only small values of $z$ $(z \approx d)$ contribute to the integral, and we obtain

$$
\begin{aligned}
W_{1}= & \int_{0}^{\infty} \frac{A_{1} r_{0}^{6} 2 R z}{45(d+z)^{9}} \mathrm{~d} z-\int_{0}^{\infty} \frac{A_{1} r_{0}^{6} 2 R z}{45(d+z+l)^{9}} \mathrm{~d} z+\int_{0}^{\infty} \frac{2 A_{1} R z}{6(d+z+l)^{3}} \mathrm{~d} z \\
& -\int_{0}^{\infty} \frac{2 A_{1} R z}{6(d+z)^{3}} \mathrm{~d} z \\
= & \frac{A_{1} r_{0}^{6} R}{1260 d^{7}}-\frac{A_{1} r_{0}^{6} R}{1260(d+l)^{7}}+\frac{A_{1} R}{6(d+l)}-\frac{A_{1} R}{6 d} .
\end{aligned}
$$

The potential function between an atom and the substrate at a distance $d$ is represented as:

$$
\Phi_{\mathrm{LJ}}=2 \pi \rho_{2}\left[\frac{C_{2} r_{0}^{6}}{90(z+d+l)^{9}}-\frac{C_{2}}{12(z+d+l)^{3}}\right],
$$

where $\rho_{2}$ is the number density of molecules in the substrate, $C_{2}$ is the interaction potential between the atom and the substrate. Thus, the potential energy between the tip and substrate is

$$
W_{2}=\int_{0}^{\infty} \frac{A_{2} r_{0}^{6} 2 R z}{45(d+z+l)^{9}} \mathrm{~d} z-\int_{0}^{\infty} \frac{2 A_{2} R z}{6(d+z+l)^{3}} \mathrm{~d} z=\frac{A_{2} r_{0}^{6} R}{1260(d+l)^{7}}-\frac{A_{2} R}{6(d+l)},
$$

where $A_{2}=\pi^{2} C_{2} \rho_{2} \mu$ is the Hamaker constant for the tip and substrate.

In Fig. 3, we set up local orthogonal coordinates $o x z$. From equations (4) and (6), the $z$ component of the interaction force between the tip and the sample can be 


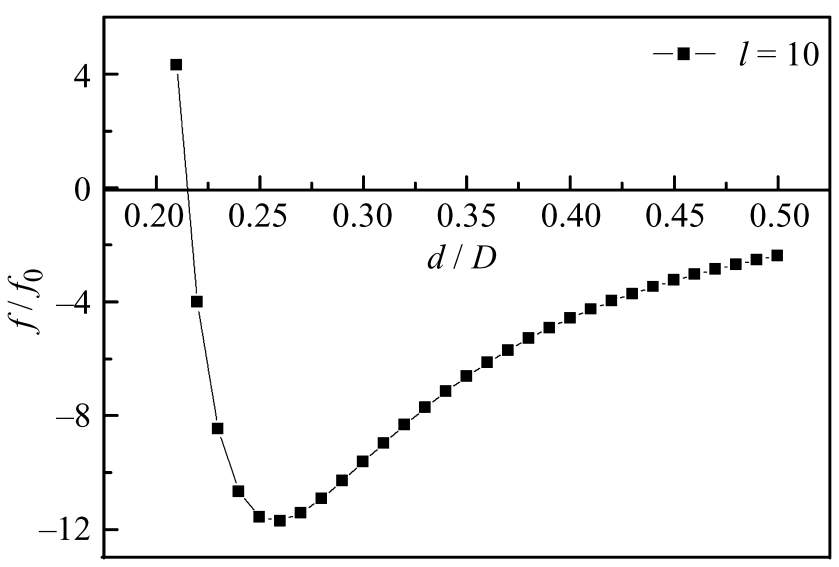

Figure 4. Dimensionless force $f / f_{0}$ versus dimensionless displacement $d / D$ with fixed chain length.

expressed by

$$
f=-\frac{\partial W_{1}}{\partial d}-\frac{\partial W_{2}}{\partial d}=-\frac{A_{1} r_{0}^{6} R}{180 d^{8}}+\frac{\left(A_{1}-A_{2}\right) r_{0}^{6} R}{180(d+l)^{8}}-\frac{\left(A_{1}-A_{2}\right) R}{6(d+l)^{2}}+\frac{A_{1} R}{6 d^{2}} .
$$

Introducing $f_{0}=\frac{A R}{6 D^{2}}$, equation (7) can be made dimensionless as follows:

$$
\frac{f}{f_{0}}=-\frac{A_{1}}{A} \cdot \frac{r_{0}^{6} D^{2}}{30 d^{8}}+\frac{\left(A_{1}-A_{2}\right)}{A} \cdot \frac{r_{0}^{6} D^{2}}{30(d+l)^{8}}-\frac{\left(A_{1}-A_{2}\right)}{A} \cdot \frac{D^{2}}{(d+l)^{2}}+\frac{A_{1}}{A} \frac{D^{2}}{d^{2}},
$$

where $f_{0}=A R / 6 D^{2}$ represents the non-retarded vdW force between a sphere of radius $R$ near a half-infinite space at separation $D$ and $A$ is the Hamaker constant. The relationship between the dimensionless $f / f_{0}$ and $d / D$ is shown in Fig. 4. We can see that the attractive force increases when the tip gradually approaches the surface, and it reaches a maximum force. Then the force decreases and when the tip is further pushed into the surface, the interaction force becomes repulsive.

In this paper, we are most interested in the point at which the corresponding force represents the adhesion force. The corresponding distance can also be calculated and is quite important. By letting $\partial f / \partial d=0$, we obtain

$$
\frac{2 A_{1} r_{0}^{6} R}{45 d^{9}}-\frac{2\left(A_{1}-A_{2}\right) r_{0}^{6} R}{45(d+l)^{9}}+\frac{\left(A_{1}-A_{2}\right) R}{3(d+l)^{3}}-\frac{A_{1} R}{3 d^{3}}=0 .
$$

Equation (9) represents the relationship between $d$ and $l$, and, thus, we can derive the value of $d$ from it which is of the order of $0.1 \mathrm{~nm}$.

\section{RESULTS AND DISCUSSION}

By substituting equation (9) into equation (8), we obtain the expression for $f / f_{0}$ without $d$, which represents the relationship between the adhesion force and the chain length of the SAMs. A decrease of the adhesion force is found as the chain 


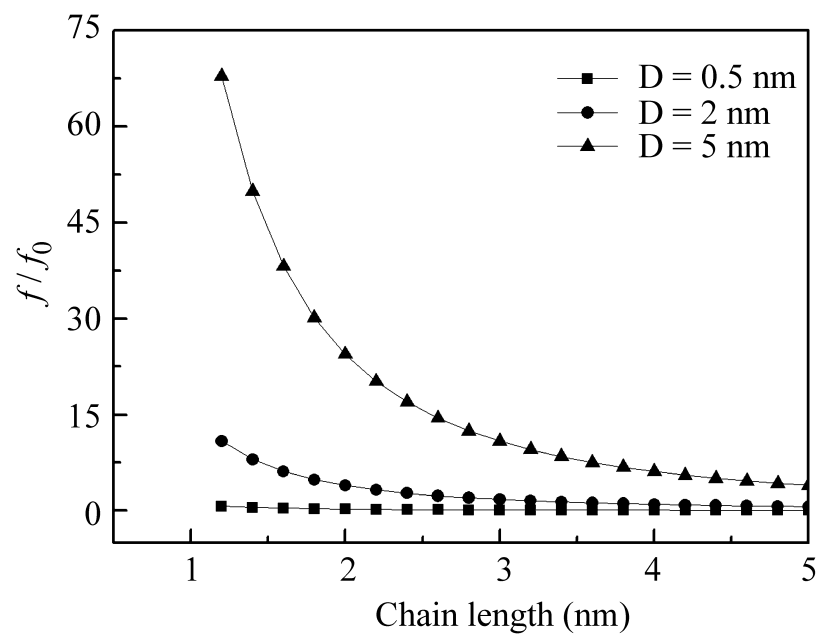

Figure 5. Behavior of the dimensionless force $f / f_{0}$ versus chain length $l$ for varying $D$.

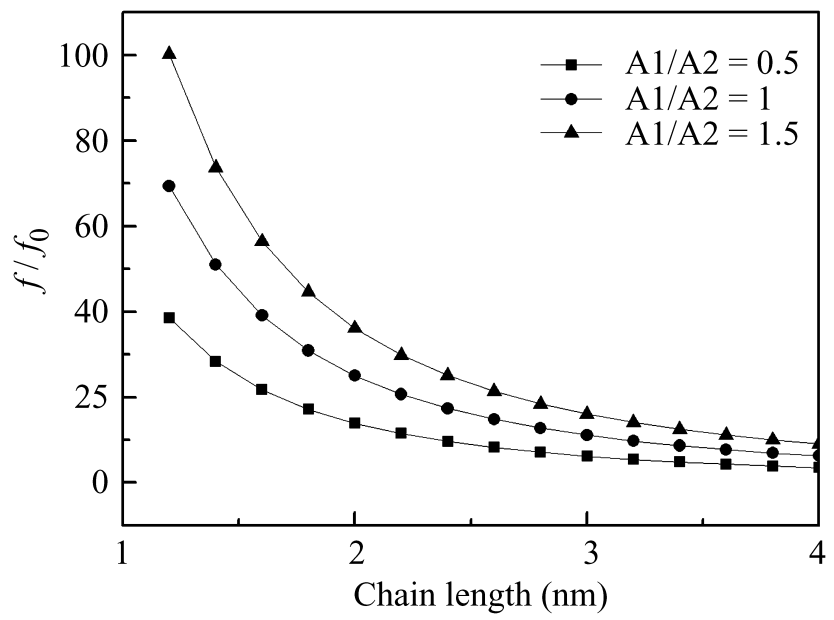

Figure 6. Behavior of the dimensionless force $f / f_{0}$ versus chain length $l$ for different ratios of Hamaker constant.

length is increased, as shown in Figs 5 and 6. It is convenient to draw these curves by making the equation dimensionless, because we need not take into account the value of Hamaker constant.

In fact, the influence of nearby atoms on the pair of interacting atoms is neglected by assuming the additivity of the vdW force and thus large errors are introduced in the calculation [25]. So we follow the Lifshitz theory that the total vdW potential as well as the constant $C$ in Eq.(1) can be written approximately as [25]:

$$
C=-\frac{3 k_{\mathrm{B}} T}{\left(4 \pi \varepsilon_{0}\right)^{2} r^{6}}\left(\frac{u_{1}^{2}}{3 k_{\mathrm{B}} T}+\alpha_{01}\right)\left(\frac{u_{2}^{2}}{3 k_{\mathrm{B}} T}+\alpha_{02}\right)-\frac{3}{2} \frac{\alpha_{01} \alpha_{02}}{\left(4 \pi \varepsilon_{0}\right)^{2} r^{6}} \frac{h v_{1} \nu_{2}}{\left(v_{1}+v_{2}\right)},
$$


where $u_{1}$ and $u_{2}$ are the dipole moments of the molecules, $k_{\mathrm{B}}$ is the Boltzmann constant, $T$ is the temperature, $\varepsilon_{0}$ is permittivity of free space, $\alpha_{01}$ and $\alpha_{02}$ are the electronic polarizabilities of the molecules, $h$ is the Planck constant, and $v_{1}$ and $\nu_{2}$ are the orbiting frequencies of the electrons [25, 29]. The first term on the right hand side of equation (10) contains the Keesom and Debye energies, which act only between polar molecules and is called polar or entropic contribution. The second term is called dispersion contribution and it acts between every molecule. The Hamaker constant can be expressed as [25]:

$$
\begin{aligned}
A_{\mathrm{H}}= & A_{\mathrm{H}, v=0}+A_{\mathrm{H}, v>0} \\
\cong & \frac{3}{4} k_{\mathrm{B}} T \frac{\varepsilon_{1}-\varepsilon_{3}}{\varepsilon_{1}+\varepsilon_{3}} \frac{\varepsilon_{2}-\varepsilon_{3}}{\varepsilon_{2}+\varepsilon_{3}} \\
& +\frac{3 h v_{e}}{8 \sqrt{2}} \frac{\left(n_{1}^{2}-n_{3}^{2}\right)\left(n_{2}^{2}-n_{3}^{2}\right)}{\sqrt{\left|n_{1}^{2}-n_{3}^{2}\right|} \sqrt{\left|n_{2}^{2}-n_{3}^{2}\right|}\left[\sqrt{\left|n_{1}^{2}-n_{3}^{2}\right|}+\sqrt{\left|n_{2}^{2}-n_{3}^{2}\right|}\right]},
\end{aligned}
$$

where $v_{e}$ is the mean absorption frequency, and $\varepsilon_{i}$ and $n_{i}$ are the static dielectric constants and refractive indices of tip, sample, and intervening medium respectively. Then we can calculate the real adhesion force by equations (7) and (9).

For silicon tip and the mica substrate, using equation (11), we obtain the Hamaker constant as follows (all the refractive index and dielectric response data are from $[29,30])$

$$
\begin{aligned}
& A_{\mathrm{H}, v=0} \approx 0.085 \times 10^{-20} \mathrm{~J}, \quad \text { at } 300 \mathrm{~K} \\
& A_{\mathrm{H}, v>0} \approx 2.48 \times 10^{-19} \mathrm{~J},
\end{aligned}
$$

giving a total value for $A_{\mathrm{H}}$ of $(0.0085+2.48) 10^{-19} \approx 2.49 \times 10^{-19} \mathrm{~J}$.

For hydrocarbons, treating them as an assembly of $\mathrm{CH}_{2}$ groups, we obtain the Hamaker constant between the AFM tip and SAMs as

$$
\begin{aligned}
& A_{\mathrm{H}, v=0} \approx 0.091 \times 10^{-20} \mathrm{~J}, \quad \text { at } 300 \mathrm{~K} \\
& A_{\mathrm{H}, v>0} \approx 4.38 \times 10^{-19} \mathrm{~J},
\end{aligned}
$$

giving a total value for $A_{\mathrm{H}}$ of $(0.0091+4.38) 10^{-19} \approx 4.39 \times 10^{-19} \mathrm{~J}$. Then we can calculate the value of the adhesion force and obtain the real force curve as shown in Fig. 7. As Fig. 7 shows, the adhesion force decreases as the chain length is increased. When the radius of curvature of the tip is changed, the adhesion force will have a small increment, but there is no effect on the general tendency of the curve, which indicates that the size of the tip does not influence the adhesion force. The adhesion force in Fig. 7 is of the order of nano-Newton, which is similar to MoralesCruz's result [31], and so it is in agreement with the actual value. When the chain length varies from $1 \mathrm{~nm}$ to $2.5 \mathrm{~nm}$, the adhesion force shows a sharp drop, and in the range of 2.5-3.5 nm the adhesion force is not sensitive to changes in chain length. From this we can see that without SAMs, the adhesion force between the AFM tip and the substrate is quite large, and when SAMs are formed on the substrate, 


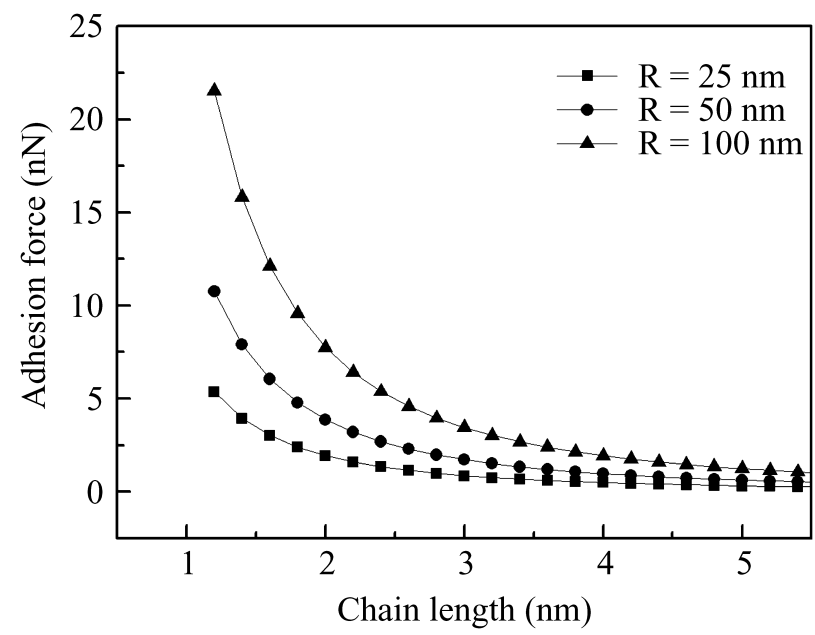

Figure 7. Adhesion force versus chain length curve for silicon nitride tip and mica substrate for different tip radii.

Table 1.

Data used in the calculation of $A_{\mathrm{H}}$

\begin{tabular}{lcl}
\hline Material & Dielectric constant $(\varepsilon)$ & Refractive index $(n)$ \\
\hline Silicon & 11.6 & 3.38 \\
Mica & 7.0 & 1.60 \\
Hydrocarbon & 2.25 & 1.50 \\
Silicon nitride & 7.4 & 1.99 \\
\hline
\end{tabular}

the adhesion force decreases dramatically. As the chain length is increased, the adhesion force decreases. Several studies have reported the effect of the chain length of molecules of a monolayer on the adhesion force [32,33]. Generally, a decrease of the adhesion force is found as the chain length is increased [21], and our results are in agreement.

Since $h v_{\mathrm{e}} \gg k_{\mathrm{B}} T$ we find that the $v>0$ dispersion force contribution is usually greater than the $v=0$ dipolar contribution. So we may use the dispersion contribution only. The corresponding data and Hamaker constants are given in Tables 1 and 2. Then we can obtain the adhesion force for different materials as Fig. 8 shows. The curve falls systematically, roughly with the square root of molecular length, which is similar to Kendall's results [34].

When considering the adhesion force between two solid surfaces or particles in air or in a liquid, it is easy to overlook or underestimate the importance role of capillary forces. The presence of capillary force can overwhelm the details of adhesion. To eliminate capillary force, our model is discussed in the situation of liquid environments or ultrahigh vacuum. And thus we can just focus on the effect 
Table 2.

Values of $A_{\mathrm{H}}$ calculated using equation (11)

\begin{tabular}{ll}
\hline System & $A_{\mathrm{H}}\left(10^{-19} \mathrm{~J}\right)$ \\
\hline Silicon/hydrocarbon/mica & 2.49 \\
Silicon/vacuum/hydrocarbon & 4.38 \\
Silicon nitride/hydrocarbon/mica & 2.06 \\
Silicon nitride/hydrocarbon/silicon & 4.82 \\
Silicon nitride/vacuum/hydrocarbon & 3.57 \\
\hline
\end{tabular}

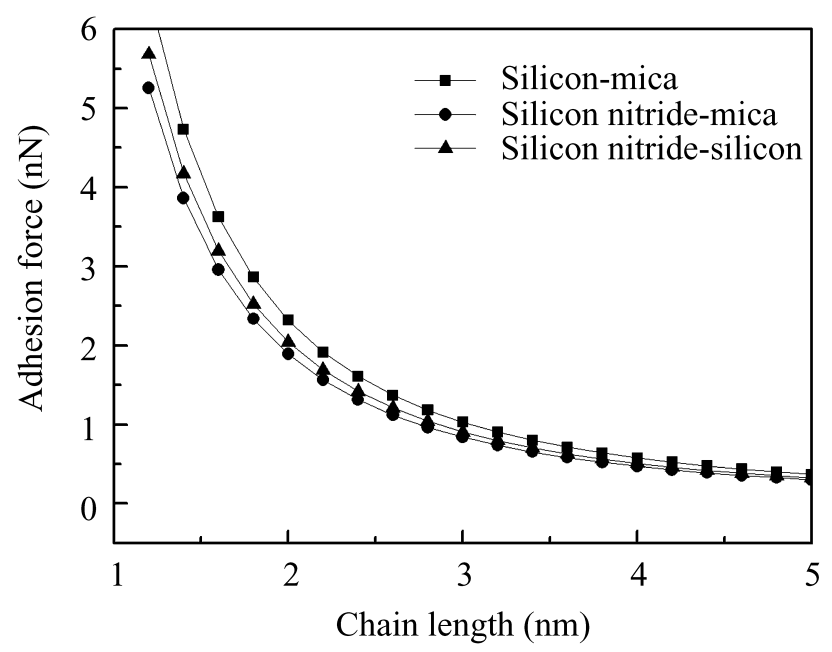

Figure 8. Adhesion force versus chain length curves for different materials.

of chain length on adhesion force. All the analyses show that the chain length of the SAMs greatly influences the adhesion force.

\section{CONCLUSIONS}

A quasi-continuum model is proposed to calculate the adhesion force between the AFM tip and SAMs on a substrate by integrating the L-J potential for the molecular interactions. According to our theoretical model, the adhesion force decreases with the increase of chain length of SAMs, which agrees well with the experimental results. Hence, a layer of SAMs formed on the substrate leads to low adhesion and thus can serve as anti-stiction coating in MEMS.

\section{Acknowledgements}

This work was supported by the Distinguished Young Scholar Fund of The National Natural Science Foundation of China (NSFC, Grant No. 10225209), a key project of Chinese Academy of Sciences (Grant No. KJCX-SW-L2). 


\section{REFERENCES}

1. R. Maboudian and C. Carraro, Annu. Rev. Phys. Chem. 55, 35 (2004).

2. Y.-P. Zhao, L. S. Wang and T. X. Yu, J. Adhesion Sci. Technol. 17, 519 (2003).

3. F. Schreiber, J. Phys.: Condensed Matter 16, 881 (2004).

4. V. V. Tsukruk, Adv. Mater. 13, 95 (2001).

5. A. Ulman, Chem. Rev. 96,1533 (1996).

6. A. Ulman, MRS Bull. 6, 46 (1995).

7. K. Komvopoulos, J. Adhesion Sci. Technol. 17, 477 (2003).

8. Z. Rymuza, Microsyst. Technol. 5, 173 (1999).

9. R. Maboudian, W. R. Ashurst and C. Carraro, Sensors Actuat. A82, 219 (2000).

10. B. Bhushan (Ed.), Springer Handbook of Nanotechnology. Springer, Berlin (2004).

11. A. Ulman, An Introduction to Organic Ultra Thin Films: From Langmuir to Self-Assembly. Academic Press, Boston, MA (1991).

12. X. D. Xiao, J. Hu, D. H. Charych and M. Salmeron, Langmuir 12, 235 (1996).

13. H. I. Kim and J. E. Houston, J. Am. Chem. Soc. 122, 12045 (2000).

14. S. Lee, A. Puck, M. Graupe, G. R. Colorado, Y. S. Shon, R. Lee and S. S. Perry, Langmuir 17, 7364 (2001).

15. S. L. Ren, S. R. Yang and Y.-P. Zhao, Langmuir 20, 3601 (2004).

16. S. L. Ren, S. R. Yang, J. Q. Wang, W. M. Liu and Y.-P. Zhao, Chem. Mater. 16, 428 (2004).

17. S. L. Ren, S. R. Yang and Y.-P. Zhao, Langmuir 19, 2763 (2003).

18. C. D. Frisbie, L. F. Rozanyal, A. Noy, M. Wrighton and C. M. Lieber, Science 265, 2071 (1994).

19. B. Bhushan and H. W. Liu, Phys. Rev. B63, 245412 (2001).

20. E. W. van der Vegte, S. Andrei and H. Georges, Langmuir 16, 3249 (2000).

21. A. Noy, D. Frisbie, L. F. Rozsnyai, M. S. Wrighton and C. M. Lieber, J. Am. Chem. Soc. 117, 7943 (1995).

22. M. Chandross, G. S. Grest and M. J. Sterens, Langmuir 18, 8392 (2002).

23. N. J. Brewer, B.D. Beake and G. J. Leggett, Langmuir 17, 1970 (2001).

24. M. Nakano, T. Ishida, T. Numata, Y. Ando and S. Sasaki, Jpn. J. Appl. Phys. 42, 4734 (2003).

25. H. J. Butt, B. Cappella and M. Kappl, Surface Sci. Rep. 59, 1 (2005).

26. Z. Wei and Y.-P. Zhao, Chin. Phys. 13, 1320 (2004).

27. X. D. Xiao and L. M. Qian, Langmuir 16, 8153 (2000).

28. G. Barbero and L. R. Evangelista, Phys. Rev. E70, 041407 (2004).

29. J. N. Israelachvili, Intermolecular and Surface Forces, 2nd edn. Academic Press, London (1985).

30. T. J. Senden and C. J. Drummond, Colloids Surfaces A94, 29 (1995).

31. A. L. Morales-Cruz, R. Tremont, R. Martinez, R. Romanach and C. R. Cabrera, Appl. Surface Sci. 241, 371 (2005).

32. W. Barger, D. Koleske, K. Feldman, D. Kruger and R. Colton, J. Am. Chem. Soc. 212, 374 (1996).

33. G. Bar, S. Rubin, A. N. Parikh, B. I. Swanson, T. A. Zawodzinski and M. H. Whangbo, Langmuir 13, 373 (1997).

34. K. Kendall, Molecular Adhesion and Its Applications. Kluwer Academic/Plenum, New York, NY (2001).

\section{APPENDIX A. NOMENCLATURE}
$A_{1}, A_{2}, A, A_{\mathrm{H}}$
Hamaker constants
$H$
Planck constant 


$\begin{array}{ll}k_{\mathrm{B}} & \begin{array}{l}\text { Boltzmann constant } \\ l\end{array} \\ n_{1}, n_{2}, n_{3} & \begin{array}{l}\text { length of SAM molecules } \\ \text { refractive indices }\end{array} \\ T & \text { curvature radius of the tip } \\ u_{1}, u_{2} & \text { temperature } \\ \alpha_{01}, \alpha_{02} & \text { dipole moments of molecules } \\ \varepsilon_{0} & \text { electronic polarizabilities of molecules } \\ \varepsilon_{1}, \varepsilon_{2}, \varepsilon_{3} & \text { static dielectric constants } \\ \mu & \text { density of molecules of the tip } \\ \rho_{1}, \rho_{2} & \text { number densities of molecules } \\ v_{1}, v_{2} & \text { orbiting frequencies of electron } \\ v_{\mathrm{e}} & \text { mean absorption frequency }\end{array}$

\section{APPENDIX B. DERIVATION OF EQUATIONS (2) AND (5)}

The interparticle potential is modeled by the $\mathrm{L}-\mathrm{J}$ potential of the form

$$
e(r)=\frac{C r_{0}^{6}}{r^{12}}-\frac{C}{r^{6}} .
$$

The interaction potential energy between an atom of the tip and the SAMs at a distance $z$ is made up of the sum of its interactions with all the molecules in the SAMs. As Fig. B-1 shows, for molecules in a circular ring of cross-sectional area $\mathrm{d} x \mathrm{~d} z$ and radius $x$, the ring volume is $2 \pi x \mathrm{~d} x \mathrm{~d} z$, and the number of molecules in the ring will be $2 \pi \rho_{1} x \mathrm{~d} x \mathrm{~d} z$, where $\rho_{1}$ is the number density of molecules in the SAMs. The net interaction energy for a molecule at a distance $Z$ away from the

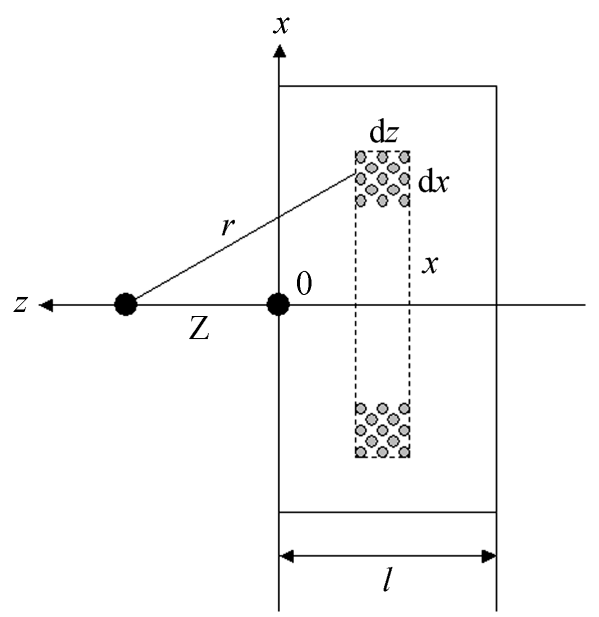

Figure B-1. Model for integrating the interaction energies between a molecule and SAMs. 
surface will, therefore, be

$$
\begin{aligned}
\Phi_{\mathrm{LJ}} & =\int_{Z}^{Z+l} 2 \pi \rho_{1} \int_{0}^{\infty}\left[\frac{C_{1} r_{0}^{6}}{\left(x^{2}+z^{2}\right)^{6}}-\frac{C_{1}}{\left(x^{2}+z^{2}\right)^{3}}\right] x \mathrm{~d} x \mathrm{~d} z \\
& =\int_{Z}^{Z+l} 2 \pi \rho_{1}\left[\frac{C_{1} r_{0}^{6}}{10 z^{10}}-\frac{C_{1}}{4 z^{4}}\right] \mathrm{d} z \\
& =2 \pi \rho_{1}\left[\frac{C_{1} r_{0}^{6}}{90 z^{9}}-\frac{C_{1} r_{0}^{6}}{90(z+l)^{9}}+\frac{C_{1}}{12(z+l)^{3}}-\frac{C_{1}}{12 z^{3}}\right],
\end{aligned}
$$

and the interaction potential energy between an atom and the substrate at distance $z$ is expressed as follows

$$
\begin{aligned}
\Phi_{\mathrm{LJ}} & =\int_{0}^{\infty} 2 \pi \rho_{2} \int_{0}^{\infty}\left[\frac{C_{2} r_{0}^{6}}{\left(x^{2}+z^{2}\right)^{6}}-\frac{C_{2}}{\left(x^{2}+z^{2}\right)^{3}}\right] x \mathrm{~d} x \mathrm{~d} z \\
& =\int_{0}^{\infty} 2 \pi \rho_{2}\left[\frac{C_{2} r_{0}^{6}}{10 z^{10}}-\frac{C_{2}}{4 z^{4}}\right] \mathrm{d} z \\
& =2 \pi \rho_{2}\left[\frac{C_{2} r_{0}^{6}}{90(z+d+l)^{9}}-\frac{C_{2}}{12(z+d+l)^{3}}\right] .
\end{aligned}
$$

